\title{
Links between Adolescents' Health Literacy and their Physical Activity and Body Mass Index
}

\author{
Renata Rutkauskaitė, Konsta Kuusinen \\ Lithuanian Sports University, Kaunas, Lithuania
}

\begin{abstract}
Background. The aim of the study was to identify links between health literacy, health information literacy and physical activity as well as body mass index in adolescents.

Methods. The study was carried out from September till the end of November, 2018. Research participants were 167 14-18-year-old students from Kaunas, Klaipeda and Vilnius ( $n=107$ females, $n=60$ males). Participants were asked to fill in an anonymous online questionnaire, which consisted of basic demographics, physical activity, health information literacy and health literacy. Adolescents' body mass index was calculated using ISO-BMI calculations. Physical activity was assessed by Petronyte's physical activity questionnaire. To determine adolescents' everyday health information literacy, we used self-assessed 10-item screening tool (EHIL-10) and to determine health literacy, we used Newest Vital Sign (NVS) screening test. Data analysis was performed using SPSS 23.0 program for Windows.

Results. Health literacy did not have association with body mass index or the level of physical activity. Health literacy scores gave important data of the level of health literacy. The scores indicated that $21.6 \%$ of Lithuanian adolescents had adequate level of health literacy. It was found that age correlated positively with health literacy. Selfassessed everyday health information literacy was higher since $85 \%$ of the students had medium high or high level of health information literacy. The data revealed that adolescents were not sure where they could find health related information and who they could trust in health-related issues. For males, finding health information and knowing who they could trust in health issues was easier than for females. Health information literacy did not have association with ISO-BMI. Sufficient evaluation of health-related information was higher among normally weighted adolescents. Physically active students had higher health information literacy and participation in sports club activities had positive relation with better health information literacy. Additionally, involvement in organized activities associated with superior understanding of terms and sentences of health information. Evaluation of health information was higher among those who were actively involved in organized physical activities.

Conclusions. Health information literacy did not have association with ISO-BMI. Those, who were involved in sports club activities or were more actively involved in organized physical activities had better health information literacy and evaluation of health information. Nevertheless, there was no connection between health literacy and physical activity.
\end{abstract}

Keywords: health literacy, health information literacy, physical activity, body mass index.

\section{INTRODUCTION}

$\mathrm{I}$ $\mathrm{t}$ is difficult to avoid health information in the 21 st century. Nowadays societies encounter health decision-making paradox. Individuals are challenged to make healthy lifestyle choices on a daily basis. Today's societies are constructed by unhealthy lifestyle marketing and difficult health care systems. Education systems are not strong enough to offer skills to improve individual health (World Health Organization, 2017).

Even a society where health information is increasing, health literacy has been measured as inadequate. In a 2018 survey in Lithuania, less than half of Lithuanian young adults had sufficient or excellent health literacy. This is important 
for maintaining good health and quality of life (Cesnaviciene, Kalinkeviciene, \& Ustilaite, 2018). Inadequate or limited health literacy can be seen as unhealthy choices, risk-taking behaviour, bad health and less self-control (World Health Organization, 2017). Also, inadequate health literacy is related to higher morbidity (Baker et al., 2007; Bostock \& Steptoe, 2012) and multi-morbidity (Heijmans, Waverijn, Rademakers, van der Vaart, \& Rijken, 2015). This problem is evident not only in Lithuania, but also around the world. For example, in Europe, almost half of European adults in eight countries have been found to demonstrate insufficient or problematic health literacy (Sørensen et al., 2015; World Health Organization, 2017).

The most common and WHO approved Health Literacy (HL) definition describes it as knowledge, motivation and competences to access, understand, appraise and apply information to make decisions in terms of health care, disease prevention and health promotion (Sørensen et al., 2015). These are involved in everyday life in order to think critically and make decisions in maintaining or improving quality of life gradually (World Health Organization, 2017).

Despite the complexity of HL and its unstable definition, it was possible to measure HL. Measurements were developed in the U.S. Most of the assessment tools measured quantitative HL, but qualitative studies were also performed (Rask, 2012). Everyday health information challenges are not widely measured. In this research, this aspect aimed to show a wider perspective of participants' HL levels.

Health literacy (HL) has been evaluated around the world, but studies have focused primarily on adult population in the context of health care (Rask, 2012). This encourages widening research to children and adolescents. A study by Davis et al. (2006) tried to validate Rapid Estimate of Adolescents' Health Literacy (REALM-Teen). They found that this test was valid for brief screening HL. However, other researchers criticized this method since it relied on written understanding and did not measure HL by communication abilities or critical thinking skills (Manganello, 2007; Nielsen-Bohlman et al., 2004).

In children, high health literacy is related to less obesity (Shih, Liu, Liao, \& Osborne, 2016), but Chari, Warsh, Ketterer, Hossain, and Sharif (2014) found that children's obesity was related to parental obesity and parental health literacy (Marks, 2015). In adolescents, obesity is strictly proportional to HL (Chari et al., 2014). A survey with a larger sample produced similar results. A total of 1035 respondents demonstrated that low HL was significantly associated to overweight and obesity in adolescents. Therefore, the aim of the research was to identify health literacy and health information literacy links to physical activity levels and body mass index in adolescents. Also, in this research we tried to find if active participation in organized activities (physical education and sports club activities) had an effect on the levels of health literacy. The hypothesis was that high BMI and low physical activity levels in adolescents might be related to poor health literacy and low health information literacy in 14-18-year-old adolescents. In this research we measured the health status, self-assessed everyday health literacy and health information literacy of 14-18-year-old adolescents.

\section{METHODS}

The aim of the study was to identify the links between health literacy, health information literacy and physical activity levels as well as body mass index in adolescents. This research is a quantitative study. Research was carried out in autumn 2018 using an anonymous online questionnaire. The data was collected from upper secondary schools of Kaunas, Vilnius and Klaipedda regions in Lithuania.

In this sample, we followed the age criterion. Ages had to range from 14 to 18 years. Six of the respondents did not meet this criterion, and they were excluded from the study; 167 responses met the age criterion. In terms of gender, females made up $64.1 \%(n=107)$ and males $35.9 \%(n=60)$ of the 167 participants. The years of birth were divided into five classes: $2000(14.4 \%, n=24), 2001(21.6 \%$, $n=36), 2002(19.8 \%, n=33), 2003(40.1 \%, n=$ $67)$ and $2004(4.2 \%, n=7)$. There were first year students $44.3 \%(n=74)$, second year students $20.4 \%(n=34)$, third year students $21.6 \%(n=36)$ and fourth year students $13.8 \%(n=23)$. Of all the respondents, $5.4 \%(n=9)$ had dyslexia diagnosed by an expert.

The questionnaire was shared online for 60 Lithuanian gymnasiums. We colected 173 responses to the online questionnaire in English. The questionnaire included basic demographics about respondents' gender, year of birth, class rank/ years of studies, height and weight and diagnosis of dyslexia. Height and weight were used for calculating participants' ISO-BMI to evaluate their 
health status. Dyslexia was asked about as it might have had an effect on the answers. Other evaluation parts were PA, everyday health information literacy and HL.

Health literacy. Newest Vital Sign (NVS) is a quick and accurate screening test for limited HL. NVS was chosen because it was not only reliable in primary health care setting for adults, but also among adolescence population (Caldwell, Carter, Becker, \& Mackert, 2018). The NVS evaluated both literacy and numeracy (the ability to understand and work with numbers) as well as the ability to locate and apply information. NVS is constructed by nutrition label and followed by six questions. Participants read given nutrition label and then answered the questions, which demonstrated their ability to apply the given information. The answers are evaluated as correct or incorrect. Each correct answer on the NVS was one point. The total scores of NVS ranged from 0 to 6 . In this sample the result covered the scale from the lowest score (0) to the highest score (6). On NVS, limited HL was $<4$ points and adequate HL was $>4$ points. Participants who scored $<2$ points had inadequate HL. In this research, participants were divided into three groups by their HL level evaluated by NVS: inadequate, limited and adequate HL. The lowest possible score was one point and the highest score could be six points (Weiss et al., 2005). NVS test results were compared with the results of EHIL-10. We presume that inadequate health literacy was associated with low or medium low EHIL.

Health information literacy. Niemela et al. (2012) developed a ten-item screening tool for measuring everyday health information literacy (EHIL) and tested it among Finnish students, and based on the results they suggested a threefactor structure for it (motivation, confidence, and evaluation). The tool was created by applying the Medical Library Association's definition of HIL. It aimed to detect individuals' problems related to their interest, motivation, finding, understanding, evaluating and using health information and being literate at the average level. The item was constructed using ten items to which the participants were instructed to respond on a scale from one (strongly disagree) to five (strongly agree).

The first item asked about the need of health information and perceived importance of health information. The second was about the respondent's ability to search health information. Motivation and independence to make health related decisions were checked in item 3. Items 4-7 assessed the evaluation of health information sources from printed materials and web sources. Item 8 assessed the respondent's difficulties in understanding health terminology and written information of health information. The ninth item was about the use of health information to make proper health decisions. The last items asked, if the respondent found trust in people about their health issues (Niemela et al., 2012).

In addition, two more questions were asked. In the first question, the respondent was asked to mention 3-5 things which the respondent used in the evaluation of the reliability of health-related information on the Internet. The second question was about the issues of searched health information. The following options were given: A) Nutrition, B) Physical exercise, C) Intoxicants, D) Vaccination, E) Medication, F) Mental health, G) Sexual health, H) Symptoms, I) Own sickness, J) Others' sickness, and $\mathrm{K}$ ) Something else, what? (Niemela et al., 2012).

EHIL-10 measured three factors: 1) motivation, 2) confidence and 3) evaluation. The assessments 1 , 2, 3 and 9 collected information about motivation. These questions were about the interest of health information, the knowledge of relevant sources and readiness to apply health information. Assessments 4, 5 and 10 were about confidence. They measured participants' ability to find health related information and their trust or belief in health-related information. Assessments 6 and 7 measured the factor evaluation. These two statements were about printed health information materials and web materials. Statement 8 was an independent statement which did not belong to any of these three factors. It gave information about difficulties in understanding terminology and relevant information about HL levels and could not be used in statistical analysis in comparison with other factors (Niemela et al., 2012).

EHIL1-9 was evaluated by scores ranging from 9 points till 45 points, where 9 points was the lowest possible score and 45 points was the highest possible score. EHIL 1, 2, 3, 6, 7 and 9 were summed up on a scale from one to five and EHIL 4, 5 and 10 were summed with reversed values from one to five. Eighth statement were not calculated in this scale. Participants were divided into four categories by their everyday health information level: low (9-18 points), medium low (18-27 points), medium high (27-36 points) and high 
(36-45 points). In EHIL1-9, we measured factors of motivation, confidence and evaluation. EHIL8 was an independent statement, which gave data about difficulties in understanding sentences and words about health-related information.

Physical activity. Physical activity levels were evaluated by WHO recommendations of PA levels, whether they were actively involved in sports club activities and if the respondents participated in physical education classes. The goal in evaluating PA was to find out how often the respondent was physically active in a week, and the association between measured BMI, HL and HIL. Additionally, questions about participation in physical education (PE) or sports club activities gathered data about the effect of physical education and sports club activities on HL.

Physical activity included games, sports, transportation, chores, recreation, and physical education or planned exercise in a context of family, school and community activities (WHO, 2018). Respondents were asked how many times in a week they were physically active in their leisure time (doing sports or exercise). Options were given as follows: A) Never, B) Less than once a month, C) Once a month, D) Once a week, E) 2-3 times/week, F) 4-6 times/week, G) Everyday and I) Medical disability as a barrier for physical activity. Also, they were asked how many hours in a week they were active (doing sports or exercise) in their leisure time. Options were given as following A) 0 hours, B) 0.5 hours, C) 1 hour, D) 2-3 hours, E) 4 - hours, F) 7 and more hours. Participants were divided into two groups: active and inactive according to the duration and frequency of PA (Petronyte, 2009).

Participation in PE and in sports club activities was assessed by options yes or no. Questions were given as follows: "Do you actively (multiple times/ week) participate in sports club/physical education classes? The questions were asked separately.

This did not measure PA comprehensively, but it gave enough information for this research whether PA was associated with lower HL and lower HIL and if participation in organized sports had an effect on the levels of HL.

Body mass index. Adolescents' BMI was calculated in the same way as adults' BMI. The calculated ISO-BMI was not relevant for comparison with adults' BMI because adolescents' proportions are different than those of adults and they change with age and growth. However, it is possible to detect overweight and underweight.
This calculation is valid before the age of 18 (Dunkel, Saarelma. \& Mustajoki, 2016), so it was used in this study.

If the participant's ISO-BMI was at the range of $17-25$, the participant had normal weight. BMI values 25-30 may show that the participant is tall and muscular at this age, but most likely it can be analysed as overweight as it is in this research. Values ranging from 30 to 35 mean that the participant is obese and a value more than 35 means remarkable obesity. If the value is less than 17, it means that the participant is underweight (Dunkel et al., 2016). In this research, the sample was divided into three categories by their ISO-BMI $(<25$ normal weight, 25-30 overweight and $>30$ obese).

Mathematical statistics. The data was analysed using SPSS 23.0 program for Windows. Collected data was not normally distributed. Data included outliers (Q-Q probability plots), histograms did not follow normal curve and Kolmogorov-Smirnov was $<0.05$. Therefore, in statistical analysis we used nonparametric tests: frequency analysis, comparison of means (Mann-Whitney), correlation analyses (Spearman's), analysis of variance (Kruskall-Wallis) and cross-tab analyses (chisquare). Margin of error was 5 and the confidence level was selected at $95 \%$. The data was considered as statistically significant if the meaning of $p$ value was $<.05$. The data was insignificant if $p$ value was $>.05$.

\section{RESULTS}

Physical activity. Sample population was divided into two categories by their PA level: physically inactive and physically active. Also, respondents were asked whether they were regularly involved in physical education and sports club activities. PA was measured by the frequency and amount per week. Of 167 responses, 66.5\% were physically inactive $(n=111)$ and $33.5 \%$ were physically active $(n=56)$. The frequency sample of PA did not follow normal distribution, whereas the mode was being physically active $2-3$ / week $(37.1 \%, n=62)$. For $1.2 \%$ of the respondents $(\mathrm{n}=2)$ medical disability was a barrier for physical activities. The amount of PA in a week (hours) did not follow normal distribution, whereas the mode of hours was 2-3 hours/week $(35.9 \%, n=60)$.

The proportion of physical activity and physical inactivity in terms of gender: males were more 
physically active $(51.7 \%, n=31)$ than females $(23.4 \%, n=25)$, which is statistically significant $(p=.001) ; 66.5 \%(n=111)$ of participants did not participate in sports club activities many times in a week. Of those who participated in sports club activities, $67.9 \%(n=38)$ were physically active and those whom did not participate in sports club activities, $83.8 \%(n=93)$ were physically inactive. PA was higher among those who were involved in sports club activities $(p=.001)$. In terms of gender, males were more involved in sports clubs $(48.3 \%$, $n=29)$ than females $(25.2 \%, n=27)(p=.002)$. Even $58.7 \%(n=98)$ of the participants were actively involved in PE classes. Among adolescents who participated to $\mathrm{PE}, 34.7 \%(n=34)$ were physically active. Of those who did not participate to PE, $31.9 \%(n=21)$ were physically active. There were no differences in PA levels by participation in $\operatorname{PE}(p=.705)$. No differences were found between gender and $\operatorname{PE}(p=.214)$ or participation $(p=.216)$. By the class rank, first year students were the most physically active $(40.5 \%, n=30)$.

Body mass index. The ISO-BMI was categorized into three classes: normal weight $<$ 25 , overweight $25-30$ and obese $>30$. The sample did not follow normal distribution where the mean BMI of Lithuanian adolescents was 22.0, which is in the range of normal weight (Dunkel et al., 2016). The mode was 21.0 and standard deviation 3.7616. Among the respondents, $85.0 \%(n=142)$ normally weighted, $10.2 \%(n=17)$ were overweighed and
$4.8 \%(n=8)$ were obese. In terms of gender, majority of females had normal weight $(89.7 \%, n=$ 96) and also majority of males had normal weight $(76.7 \%, n=46) ; 6.5 \%$ of girls were overweighed $(n=7)$ and obese $3.7 \%(n=4)$. Among boys, $16.7 \%$ were overweighed $(n=10)$ and $6.7 \%$ were obese $(n=4)$. Bas to the links overweight and gender, boys were more often overweighed than girls $(p=.023)$.

Comparing PA and ISO-BM, we found that normally weighted adolescents were more physically inactive $(68.3 \%, n=97)$ than physically active $(31.7 \%, n=45)$. In the group of overweight, $47.1 \%(n=8)$ were physically inactive and $52.9 \%$ $(n=9)$ were physically active. Obese adolescents were mostly physically inactive $(75 \%, n=6)$. Connection between ISO-BMI and PA was not statistically significant. Correlations between PA frequencies, hours of physical activity and ISOBMI were also not statistically significant.

Health literacy. The NVS revealed differences among students who had similar educational status and belonged to the same age group. The sample did not follow normal distribution, where the mean was 2.52, the mode was 2 and the standard deviation was 1.875 (Table1). Respondents were divided into three groups by their HL level; inadequate, limited and adequate HL. The frequency analysis showed that $54.5 \%(n=91)$ of Lithuanian adolescents had inadequate HL. Among the respondents, $24.0 \%$ $(n=40)$ had limited HL and $21.6 \%(n=36)$ had

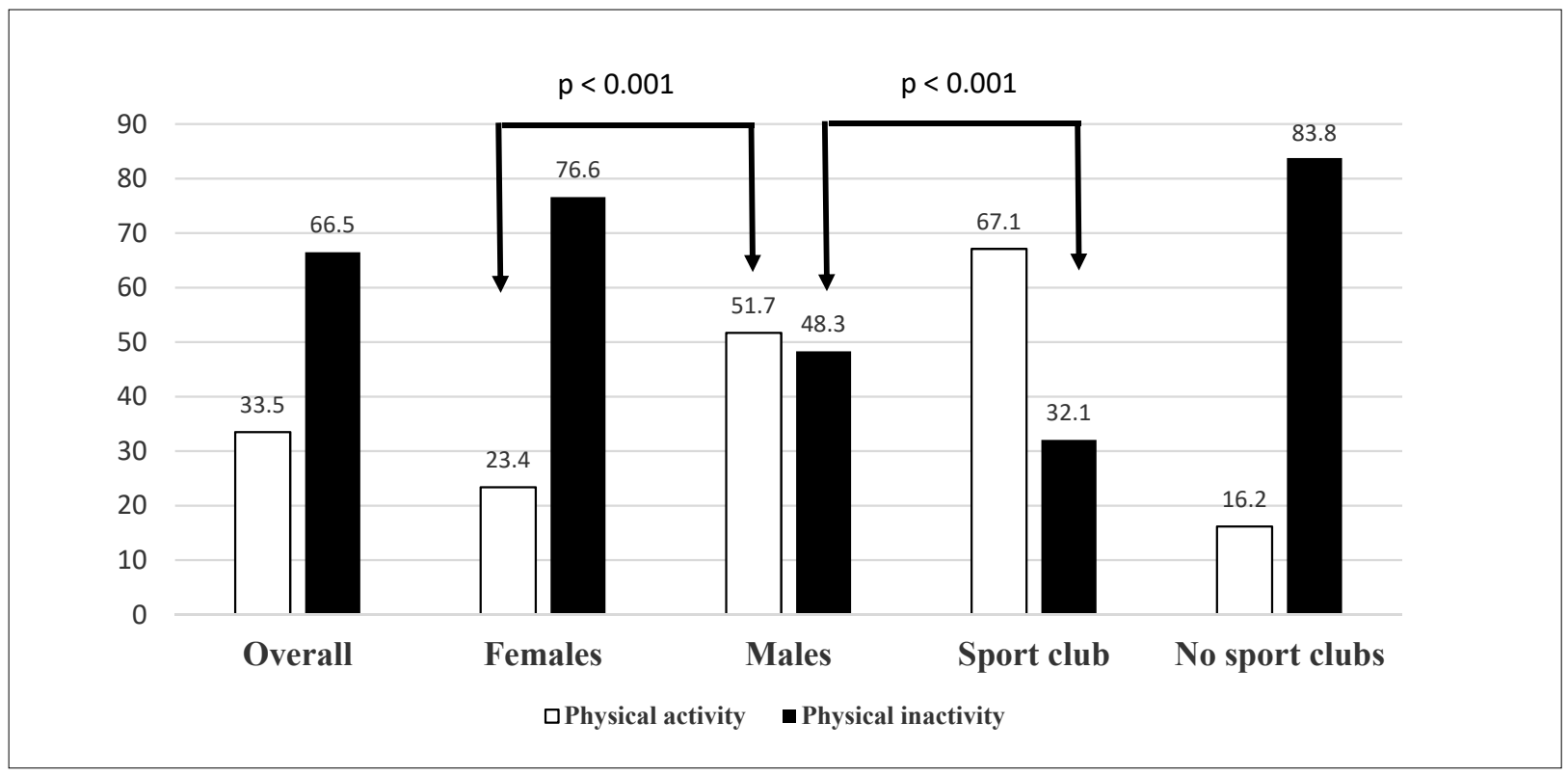

Figure 1. Physical activity differences between different groups in percentages

Note. Significant differences were found in physical activity between males and females $(p=.001)$ and participation to sport club activities $(p=.001)$. 


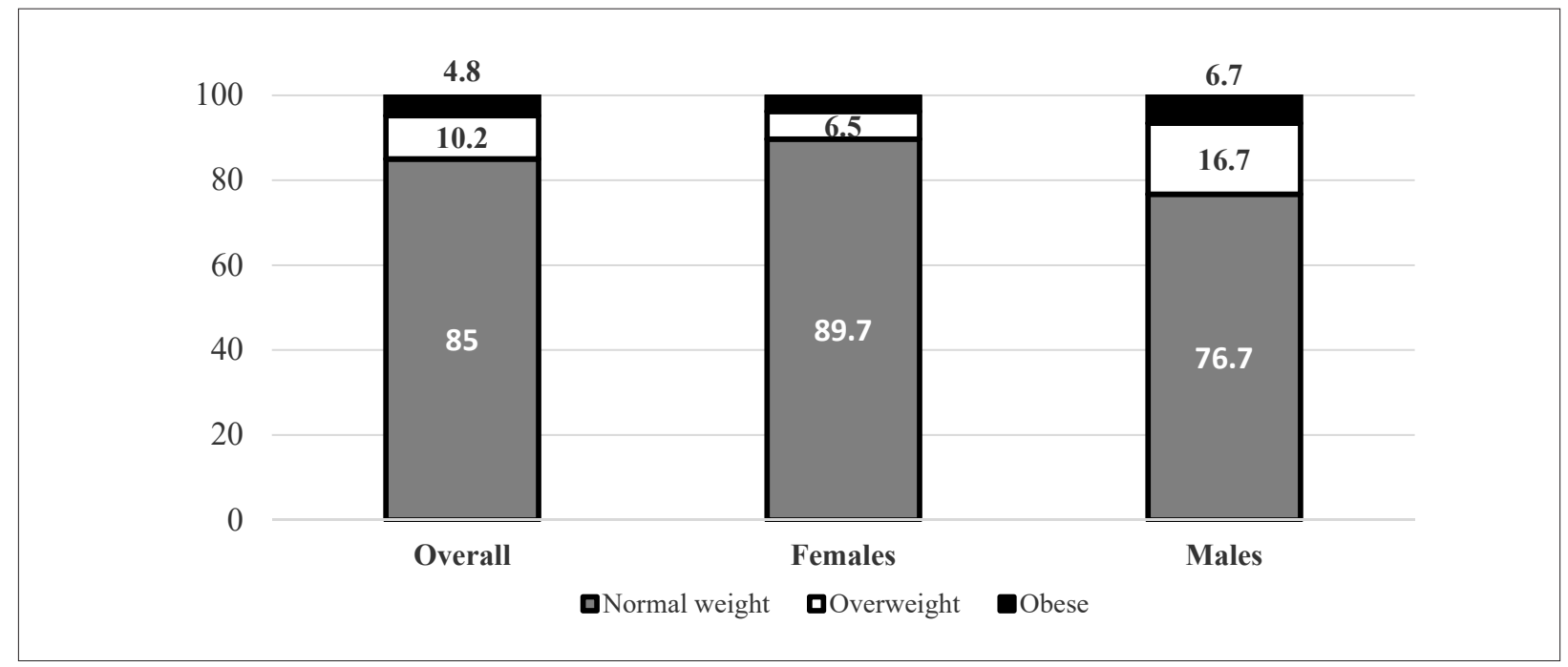

Figure 2. Bodyweight distribution by the gender

adequate HL. Gender or ISO-BMI differences in HL were not found. Females mean result of NVS was 2.58 and that of males was 2.42 on a scale from 0 to 6 . However, HL differences were found by the age and class rank. Fourth (mean 2.83) and first year (mean 2.97) students demonstrated higher HL than second (mean 2.24) and third year students (mean 1.67) ( $p=.003)$. Correlation between results in NVS and age was positive and weak $(r=0.157$, $p=.043)$. Students who had diagnosed dyslexia $89.8 \%(n=8)$ were inadequately health literate.

NVS, EHIL1-9 and EHIL8 results were compared with each other. We found no differences between HL and HIL levels ( $p=.731)$. NVS results were positively correlated with EHIL8 statement "Terms and sentences of health information are often difficult to understand" ( $r=.662, p=.034)$. Furthermore, NVS result and EHIL1-9 results had

Table 1. Newest Vital Sign (NVS) score distribution by gender, class rank, ISO-BMI and PA

\begin{tabular}{|c|c|c|c|c|}
\hline \multirow{3}{*}{ Variable } & \multicolumn{4}{|c|}{ Newest Vital Sign score } \\
\hline & Inadequate & Limited & Adequate & \multirow{2}{*}{ Mean \pm Std. deviation } \\
\hline & $n$ & $n$ & $n$ & \\
\hline Overall & $91(54.5 \%)$ & $40(24.0 \%)$ & $36(19.6 \%)$ & $2.52 \pm 1.875$ \\
\hline \multicolumn{5}{|l|}{ Gender } \\
\hline Female & $56(52.3 \%)$ & $30(28.0 \%)$ & $21(19.6 \%)$ & $2.58 \pm 1.786$ \\
\hline Male & $35(58.3 \%)$ & $10(16.7 \%)$ & $15(25.0 \%)$ & $2.42 \pm 2.036$ \\
\hline \multicolumn{5}{|c|}{ Class rank } \\
\hline $1^{\text {st }}$ year & $33(44.6 \%)$ & $22(29.7 \%)$ & $19(25.7 \%)$ & $2.97 \pm 1.728$ \\
\hline $2^{\text {nd }}$ year & $21(61.8 \%)$ & $9(26.5 \%)$ & $4(11.8 \%)$ & $2.24 \pm 1.759$ \\
\hline $3^{\text {rd }}$ year & $25(69.4 \%)$ & $7(19.4 \%)$ & $4(11.1 \%)$ & $1.67 \pm 1.757$ \\
\hline $4^{\text {th }}$ year & $12(52.5 \%)$ & $2(8.7 \%)$ & $9(39.1 \%)$ & $2.83 \pm 2.229$ \\
\hline \multicolumn{5}{|l|}{ ISO-BMI } \\
\hline Normal weight & $77(54.2 \%)$ & $34(23.9 \%)$ & $31(21.8 \%)$ & $2.53 \pm 1.874$ \\
\hline Overweight & $9(52.9 \%)$ & $5(29.4 \%)$ & $3(17.6 \%)$ & $2.41 \pm 2.093$ \\
\hline Obese & $5(62.5 \%)$ & $1(12.5 \%)$ & $2(25.0 \%)$ & $2.63 \pm 1.598$ \\
\hline \multicolumn{5}{|c|}{ PA } \\
\hline Active & $29(51.8 \%)$ & $12(12.4 \%)$ & $15(26.8 \%)$ & $2.73 \pm 1.892$ \\
\hline Inactive & $62(55.9 \%)$ & $28(25.2 \%)$ & $21(18.9 \%)$ & $2.41 \pm 1.866$ \\
\hline
\end{tabular}


a positive weak correlation $(r=.902, p=.010)$. HL was linked to HIL levels and to difficulties in understanding terms and sentences of healthrelated information.

Correlations between NVS results and ISOBMI and were not significant. In the sample, normally weighed participants had mostly inadequate HL $(54.2 \%, n=77)$. In this group, $21.8 \%$ had adequate HL $(n=31)$. Overweighed adolescents had inadequate HL as well $(52.9 \%$, $n=9)$ and $17.6 \%$ had adequate HL $(n=3)$. Among obese adolescents, $62.5 \%$ had inadequate $(n=5)$ and $25 \%$ adequate HL $(n=2)$. Normally weighed and overweighed participants' HL was similar $(p=.890)$. Normal weighed adolescents' mean NVS result was 2.53 , overweighed group's mean was 2.41 and obese group's mean was 2.63 on a scale from 0 to 6 . Connections between ISO-BMI and NVS results were not statistically significant.

Comparing PA groups (inactive and active) to the score in NVS, it was found that the results were rather similar. Physically active students' NVS mean result was 2.73 whereas physically inactive students' mean was 2.41. Statistically, the differences were not significant. HL differences were not found in comparison to PA levels. PA level and HL level were similar with each other. The group of physically active students had inadequate or limited HL $(73.2 \%, n=41)$. Though, students who had adequate HL were more often physically active than inactive, but statistically it was not significant. Also, physically inactive group had mostly inadequate or limited HL $(81.1 \%, n=90)$. Physically inactive (mean 2.41) and physically active (mean 2.73) group had similar HL by NVS results $(p=.495)$ on a scale from 0 to 6 . Adolescents' NVS results did not differ whether they participated or not actively to PE or sport club activities.

Health information literacy. The sample did not follow normal distribution, whereas the mean was 32.06 , the mode 33 and standard deviation was 4.275. In this sample the results covered a scale from the lowest score (20) to the highest score (43). The frequency analysis showed that $15.0 \%$ $(n=25)$ had medium low HIL, 69.5\% $(n=115)$ had medium high HIL and $15.6 \%(n=26)$ had high HIL. Female students' mean in EHIL1-9 was 31.75 and male students' mean was 32.62. There were no statistically significant differences between genders' HIL. Even $88.9 \%(n=8)$ of participants with diagnosed dyslexia had medium high HIL.
Factors of motivation, confidence and evaluation were compared with each other. Factors of motivation and evaluation had internal correlation $(r=.280, p=.001)$. Motivation - confidence $(p=$ $.787)$ and confidence - evaluation $(p=.789)$ were not statistically significantly connected. Additionally, factors were examined with EHIL8 statement. It was found that difficulties in understanding words and sentences of health-related information correlated with factor confidence $(r=.222, p=.004)$. Other factors' correlations were not significant.

Gender differences were not found in comparison to motivation, confidence and evaluation factors. Furthermore, males agreed more with EHIL2 statement "I know where to seek health information" than girls $(p=.015)$. The mean of male students' EHIL2 answers was 4.2, and respectively the mean of female students was 3.78 on a scale from 1 to 5. Furthermore, males disagreed more with EHIL10 statement "It is difficult to know who to believe in health issues" than females ( $p=.035)$. The mean of male students' EHIL10 answers was 2.98 , and that of female students was 3.49 on a reversed scale from 1 to 5 .

Students' results in EHIL1-9 was similar. The mean results of EHIL1-9 were at the same ranges (Table 2). Age differences were not found in comparison to motivation, confidence and evaluation factors. Interestingly, relatively high number of students did not trust health information. Among older students, $64.5 \%(n=60)$ and $71.6 \%$ $(n=53)$ of the younger students were unconfident. The difference between groups was not statistically significant.

There were no differences in EHIL results between ISO-BMI groups. A normally weighed category mean result in EHIL1-9 was 32.15 on a scale from 9 to 45 points, whereas overweighed category mean was 31.53 and obese category mean was 32.06. Interestingly, when results were compared with EHIL1-9, normal weight category medium high HIL was the most common result $(66.2 \%, n=94)$. Only $17.6 \%(n=25)$ of the normally weighed population had high HIL. Overweighed and obese population had also mostly medium high HIL (overweight $88.2 \%, n=15$ and obese $87.5 \%$, $n=7$ ). Furthermore, when students' ISO-BMI were compared with motivation, evaluation and confidence factors, it was found that proportion of good evaluation of health information was higher among normally weighed students. An association 
Renata Rutkauskaite, Konsta Kuusinen

LINKS BETWEEN ADOLESCENTS' HEALTH LITERACY AND THEIR PHYSICAL ACTIVITY AND BODY MASS INDEX

Table 2. EHIL1-9 score distribution by gender, class rank, ISO-BMI, PA, HL, PE and sports club

\begin{tabular}{|c|c|c|c|c|c|}
\hline \multirow{3}{*}{ Variable } & \multicolumn{5}{|c|}{ Everyday Health Information Literacy 1-9 } \\
\hline & \multirow{2}{*}{$\frac{\text { Medium low HIL }}{n}$} & \multirow{2}{*}{$\begin{array}{c}\text { Medium high HIL } \\
n\end{array}$} & \multirow{2}{*}{$\begin{array}{c}\text { High HIL } \\
n\end{array}$} & \multirow{2}{*}{$\begin{array}{c}\text { EHIL8 } \\
\text { Mean }\end{array}$} & \multirow{2}{*}{ Mean \pm Std. deviation } \\
\hline & & & & & \\
\hline Overall & $25(15.0 \%)$ & $116(69.5 \%)$ & $26(15.6 \%)$ & 3.09 & $32.06 \pm 4.275$ \\
\hline \multicolumn{6}{|l|}{ Gender } \\
\hline Female & $20(18.7 \%)$ & $71(66.4 \%)$ & $16(15.0 \%)$ & 3.11 & $31.75 \pm 4.642$ \\
\hline Male & $5(8.3 \%)$ & $45(75.0 \%)$ & $10(16.7 \%)$ & 3.05 & $32.62 \pm 3.494$ \\
\hline \multicolumn{6}{|l|}{ Class rank } \\
\hline $1^{\text {st }}$ year & $9(12.2 \%)$ & $50(67.6 \%)$ & $15(20.3 \%)$ & 3.04 & $32.62 \pm 4.419$ \\
\hline $2^{\text {nd }}$ year & $3(8.8 \%)$ & $30(88.2 \%)$ & $1(2.9 \%)$ & 2.94 & $31.09 \pm 3.019$ \\
\hline $3^{\text {rd }}$ year & $8(22.2 \%)$ & $21(58.3 \%)$ & $7(19.4 \%)$ & 3.14 & $32.44 \pm 4.837$ \\
\hline $4^{\text {th }}$ year & $5(21.7 \%)$ & $15(65.2 \%)$ & $3(13.9 \%)$ & 3.39 & $31.09 \pm 4.295$ \\
\hline \multicolumn{6}{|l|}{ ISO-BMI } \\
\hline Normal weight & $23(16.2 \%)$ & $94(66.2 \%)$ & $25(17.6 \%)$ & 3.06 & $32.15 \pm 4.455$ \\
\hline Overweight & $1(5.9 \%)$ & $15(88.2 \%)$ & $1(5.9 \%)$ & 3.47 & $31.53 \pm 3.044$ \\
\hline Obese & $1(12.5 \%)$ & $7(87.5 \%)$ & $0(0.0 \%)$ & 2.88 & $31.50 \pm 3.388$ \\
\hline \multicolumn{6}{|l|}{ PA } \\
\hline Active & $2(3.6 \%)$ & $45(80.4 \%)$ & $9(16.1 \%)$ & 3.04 & $32.88 \pm 3.698$ \\
\hline Inactive & $23(20.7 \%)$ & $71(64.0 \%)$ & $17(15.3 \%)$ & 3.12 & $31.65 \pm 4.498$ \\
\hline \multicolumn{6}{|l|}{ HL } \\
\hline Inadequate & $14(15.4 \%)$ & $64(70.3 \%)$ & $13(14.3 \%)$ & 3.01 & $32.23 \pm 4.193$ \\
\hline Limited & $6(15.0 \%)$ & $24(60.0 \%)$ & $10(25.0 \%)$ & 2.98 & $32.28 \pm 4.982$ \\
\hline Adequate & $5(13.9 \%)$ & $28(77.8 \%)$ & $3(8.3 \%)$ & 3.42 & $31.39 \pm 3.635$ \\
\hline \multicolumn{6}{|l|}{$\mathbf{P E}$} \\
\hline No & $15(21.7 \%)$ & $41(59.4 \%)$ & $13(18.8 \%)$ & 3.21 & $31.47 \pm 2.276$ \\
\hline Yes & $10(10.2 \%)$ & $75(76.5 \%)$ & $13(13.3 \%)$ & 2.86 & $33.23 \pm 4.059$ \\
\hline \multicolumn{6}{|l|}{ Sports club } \\
\hline No & $22(19.8 \%)$ & $74(66.7 \%)$ & $15(13.5 \%)$ & 3.20 & $31.47 \pm 4.276$ \\
\hline Yes & $3(5.4 \%)$ & $42(75.0 \%)$ & $11(19.6 \%)$ & 3.01 & $33.23 \pm 4.059$ \\
\hline
\end{tabular}

between overweight and normal weight was observed $(p=.021)$. Correlation between ISO-BMI and evaluation was negative weak $(r=-.186, p=$ .016). Connections with motivation and confidence factors were not statistically significant.

PA differences were found in comparison to EHIL1-9 results. Those who were physically active enough had better HIL scores than physically inactive participants $(p=.003)$. In comparison of HIL to PA levels, we found that students with higher HIL were more often enough physically active than students who had lower HIL $(p=.012)$. The difference of HIL between PA groups was not statistically significant. EHIL1-9 results were rather similar in physically inactive (mean 31.65) and physically active (mean 32.87 ) groups on a scale from 9 to 45. Furthermore, correlations between EHIL scores and frequency of PA were positive and weak $(r=.519, p=.001)$. Correlation between EHIL scores and hours of PA in a week was also positive weak $(r=.199, p=.010)$. In comparison of EHIL motivation, confidence and evaluation to PA, we did not find any differences. Physical activity correlated with health information literacy, but not health literacy.

The participants who were actively involved in sports club activities had higher HIL than those who were not involved $(p=.013)$. Difference in HIL between those who participated and did not participate in sports club activity was statistically significant $(p=.010)$. Similar results were found in comparison of participation in PE and EHIL1-9 
results. Those who were actively involved in PE had higher HIL scores than the group who did not participate actively in PE. Association between PE and HIL results was observed $(p=.040)$. The difference in HIL between participation and no participation in PE was not statistically significant. Those who participated in PE and sports club activities disagreed more with EHIL8 statement "Terms and sentences of health information are often difficult to understand." than those who did not. The mean of those who participated actively in PE was 2.80 , and those who did not -3.20 on a scale from one to five. The difference was not statistically significant. The mean of those who participated in sports club activities was 2.86 and of those who did not participate it was 3.21 on a scale from one to five. The difference between participation and no participation in sports club activities and EHIL8 statement was statistically significant $(p=.024)$.

Comparing evaluation factor to participation in sports club activities, it was found that students who participated in sports club activities had better evaluation of health-related information $(p=.007)$. Evaluation of HL was also higher among those who participated in PE $(p=.033)$. Motivation and confidence factors' correlations were not significant.

\section{DISCUSSION}

Functional or fundamental health literacy consists of reading, writing and numerical skills (Nutbeam, 2000; Zarcadoolas, Pleasant, \& Greer, 2005). At the individual level, health literacy can be seen as capacity to obtain process and understand health related information to make appropriate health decisions (Ratzan \& Parker, 2000). Lithuanian young adults aged 18-29 have been found to have sufficient or excellent health literacy (Cesnaviciene et al., 2018). In this research the target group was younger, but the findings were similar: $21.6 \%$ had an adequate level of health literacy. Measurements were on the functional and fundamental level of health literacy by Nutbeam's (2000) and Zarcadoolas' et al. (2005) theoretical models. These levels can be considered as the lowest level of health literacy, which in turn, is alarming and requires actions.

Higher levels of health literacy within populations can increase social benefits, for example by activating communities to address the determinants of health. Understanding this proves that health literacy is not only individual's responsibility, but that government and health systems offer proper and accessible information for different populations (WHO, 2016; Rudd, 2015). As people live in the paradox of information and misinformation, health literacy becomes important. According to WHO (2017), people with stronger health literacy skills have better health and well-being, while people with limited health literacy skills tend to riskier behaviour and poorer health. Additionally, low health literacy is associated with poorer use of health care services (Berkman, Sheridan, Donahue, Halpern, \& Crotty, 2011), higher mortality among elder populations (Baker et al., 2007; Bostock \& Steptoe, 2012) and multi-morbidity (Heijmans et al., 2015). Studies have shown that people with limited health literacy have also higher health care expenses and they are using more medical treatment services instead of preventive services (Rask, 2012). Thus, health education should be priority at all levels of educational processes (Cesnaviciene et al., 2018), because tenuous health education leads to inadequate health literacy (Nutbeam, 2000). As health literacy will be carried from adolescence to adulthood (Manganello, 2007), it is important to improve health education.

Based on reviewed literature, gender connection to health literacy and self-assessed health information literacy has been ambivalent. On the other hand, males' health literacy has been weaker than that of females (Wu et al., 2010) and males have had more dispersion on their answers (Kinnunen, 2015). Other researches have shown that genders have not correlated with health literacy (Paasche-Orlow et al., 2005; Sørensen et al., 2015). In this research gender did not have connection to health literacy or self-assessed health information literacy. When gender connections were scrutinized to separate factors and questions, females had more difficulties in finding health information and challenges with those they can trust in healthrelated issues.

In previous studies, age has been associated with health literacy as it has developed by the age (Wu et al., 2010). In Manganello's (2007) framework of adolescents' health literacy, age is seen as an individual trait which forebodes health literacy. In this research age and class rank was associated with health literacy so that age correlated with health literacy. Adolescents were in total unconfident of health information literacy, but the differences in 
health information literacy between class ranks and age were not statistically significant.

In children high health literacy is related to less obesity (Shih et al., 2016), but Chari et al. (2014) found that children's obesity was related to parental obesity and parental health literacy. Obesity in adolescents is strongly related with adolescents' health literacy (Chari et al., 2014; Olyani, Tehrani, Esmaily, Rezaii, \& Vahedian-Shahroodi, 2017). In Lithuania, less than half of young adults have adequate or excellent literacy (Cesnaviciene et al., 2018). This is not associated with the number of overweighed children and adolescents in Lithuania. It was found that $12.6 \%$ of Lithuanian children and adolescents were overweighed and $4.1 \%$ were obese, which is one of the lowest across Europe (Smetanina et al., 2015). In Lithuanian adults, $60.7 \%$ of population was overweighed and $27.6 \%$ was obese. These findings are relatively high across Europe (WHO, 2013). Hence, it may explain that inadequate health literacy in young adults may be associated with overweight later in adulthood. Still, it does not explain why children and adolescent overweight is relatively small in Lithuania, whether parental overweight and health literacy are related with children and adolescent overweight. In this research there was no significant connections between ISO-BMI and health literacy. Health information literacy connections were also statistically non-significant. Furthermore, participants in the range of normal weight had better evaluation of health-related information than overweighed participants. In addition, it was found that when evaluation of health information increases the body mass seems to decrease.

From the aspect of physical activity, adolescents' health literacy has been higher among participants who are physically active enough (Paakkari, Kokko, Villberg, Paakkari, \& Tynjälä, 2017; Suka et al., 2015). Health literacy has been found to be higher among those who participate to sport club activities (Paakkari et al., 2017). In this research, there was no significant differences between health literacy or health information literacy and physical activity. Those who were physically active enough had higher health information literacy. Interestingly, those who were actively involved in organized physical activities (sports clubs and physical education) had significantly higher health information literacy levels. They had also less difficulty in understanding terms and sentences of health-related information and better evaluation of health-related information. In Huoman's (2014) research, children who participated in some sports club activity were significantly more physically active than those who were not involved. Physical education and sports club activities are experienced as the most important sources of physical activity in children and adolescents. Inadequate physical activity is a global challenge (WHO, 2018) and clearly the position of physical education and sports clubs has changed towards important movements and educators of health, instead of being an enabler of competitive sports.

The main challenge in this research was the complexity of health literacy. Health literacy does not have consensus of its definition, and it is a multidimensional concept, which means the researchers have not come up with a concrete way to measure health literacy. Also, health literacy is more investigated in the field of illness, so there are only few measurement tools adaptable for adolescents.

\section{CONCLUSION}

Health information literacy did not have association with ISO-BMI. Those, who were involved in sports club activities or were more actively involved in organized physical activities had better health information literacy and evaluation of health information. Nevertheless, there was no connection between health literacy and physical activity.

\section{REFERENCES}

Baker, D. W., Wolf, M. S., Feinglass, J., Thompson, J. A., Gazmararian, J. A., \& Huang, J. (2007). Health literacy and mortality among elderly persons. Archives of Internal Medicine, 167(14), 1503-1509.

Berkman, N. D., Sheridan, S. L., Donahue, K. E., Halpern, D. J., \& Crotty, K. (2011). Low health literacy and health outcomes: An updated systematic review. Annals of Internal Medicine, 155(2), 97-107.
Bostock, S., Steptoe, A. (2012). Association between low functional health literacy and mortality in older adults: Longitudinal cohort study. Bmj-British Medical Journal, 344, (ARTN e16) 10.1136/bmj.e1602.

Caldwell, E. P., Carter, P., Becker, H., \& Mackert, M. (2018). The Use of the Newest Vital Sign Health Literacy Instrument in adolescents with sickle cell disease. Journal of Pediatric Oncology Nursing, 1043454218767875. 
Cesnaviciene, J., Kalinkeviciene, A., \& Ustilaite, S. (2018). Young adult health literacy on health promotion as social determinant of health: Challenges for health education practice in Lithuania. In SHS Web of Conferences, 40, 02005.

Chari, R., Warsh, J., Ketterer, T., Hossain, J., \& Sharif, I. (2014). Association between health literacy and child and adolescent obesity. Patient Education and Counselling, 94(1), 61-66.

Davis, T. C., Wolf, M. S., Arnold, C. L., Byrd, R. S., Long, S. W., Springer, T., ... \& Bocchini, J. A. (2006). Development and validation of the Rapid Estimate of Adolescent Literacy in Medicine (REALM-Teen): A tool to screen adolescents for below-grade reading in health care settings. Pediatrics, 118(6), e1707-e1714.

Dunkel, L., Saarelma, O., \& Mustajoki, P. (2016). Lasten painoindeksi (ISO-BMI).

Heijmans, M., Waverijn, G., Rademakers, J., van der Vaart, R., \& Rijken, M. (2015). Functional, communicative and critical health literacy of chronic disease patients and their importance for self-management. Patient Education and Counselling, 98(1), 41-48.

Huoman, S. (2014). Urheiluseuratoiminnan merkitys lasten ja nuorten liikuntakäyttäytymisessä.

Kinnunen, N. (2015). Itsearvioitu terveyden lukutaito 13-ja 15-vuotiailla: WHO-Koululaistutkimuksen esitutkimus 2013.

Manganello, J.A. (2007). Health literacy and adolescents: A framework and agenda for future research. Health Education Research, 23(5), 840-847.

Marks, R., (2015). Childhood obesity and parental health literacy. Advances in Obesity, Weight Management \& Control, 3(3), 191-195.

Nielsen-Bohlman, L., Panzer, A. M. \& Kindig, D. A., (Eds.). (2004). Health literacy: A prescription to end confusion. Washington, National Academies.

Niemela, R., Ek, S., Eriksson-Backa, K., \& Huotari, M. L. (2012). A screening tool for assessing everyday health information literacy. Libri, 62(2), 125-134.

Nutbeam, D. (2000). Health literacy as a public health goal: A challenge for contemporary health education and communication strategies into the 21 st century. Health Promotion International, 15(3), 259-267.

Olyani, S., Tehrani, H., Esmaily, H., Rezaii, M. M., \& Vahedian-Shahroodi, M. (2017). Assessment of health literacy with the Newest Vital Sign and its correlation with body mass index in female adolescent students. International Journal of Adolescent Medicine and Health.

Paakkari, L., Kokko, S., Villberg, J., Paakkari, O., \& Tynjälä, J. (2017). Health literacy and participation in sports club activities among adolescents. Scandinavian Journal of Public Health, 45(8), 854-860.

Paasche-Orlow, M. K., Parker, R. M., Gazmararian, J. A., Nielsen-Bohlman, L. T., \& Rudd, R. R. (2005). The prevalence of limited health literacy. Journal of General Internal Medicine, 20(2), 175-184.
Petronytė, G. (2009). Lietuvos paaugliu fizinis aktyvumas ir su juo susiję veiksniai (Doctoral dissertation). Kaunas: Kauno medicinos universitetas.

Rask, M. (2012). Lukiolaisten terveydenlukutaidon ja terveysarvostusten ilmeneminen: laadullinen sisällönanalyysi vuoden 2007 terveystiedon ylioppilaskirjoitusten reaalikokeen vastauksista.

Ratzan, S. C., Parker, R. M. (2000). Introduction. In: National Library of Medicine Current Bibliographies in Medicine: Health Literacy. National Institutes of Health, U.S. Department of Health and Human Services.

Rudd, R. E. (2015). The evolving concept of health literacy: New directions for health literacy studies. Journal of Communication in Healthcare, 8(1), 7-9. doi: 10.1179/1753806815Z.000000000105

Shih, S. F., Liu, C. H., Liao, L. L., \& Osborne, R. H. (2016). Health literacy and the determinants of obesity: A population-based survey of sixth grade school children in Taiwan. BMC Public Health, 16(1), 280.

Smetanina, N., Albaviciute, E., Babinska, V., Karinauskiene, L., Albertsson-Wikland, K., Petrauskiene, A., \& Verkauskiene, R. (2015). Prevalence of overweight/ obesity in relation to dietary habits and lifestyle among 7-17 years old children and adolescents in Lithuania. BMC Public Health, 15(1), 1001.

Sørensen, K., Pelikan, J. M., Röthlin, F., Ganahl, K., Slonska, Z., Doyle, G., ... \& Falcon, M. (2015). Health literacy in Europe: Comparative results of the European health literacy survey (HLS-EU). European Journal of Public Health, 25(6), 1053-1058.

Suka, M., Odajima, T., Okamoto, M., Sumitani, M., Igarashi, A., Ishikawa, H., ... \& Sugimori, H. (2015). Relationship between health literacy, health information access, health behavior, and health status in Japanese people. Patient Education and Counselling, 98(5), 660668.

Weiss, B. D., Mays, M. Z., Martz, W., Castro, K. M., DeWalt, D. A., Pignone, M. P., ... \& Hale, F. A. (2005). Quick assessment of literacy in primary care: The newest vital sign. The Annals of Family Medicine, 3(6), 514522.

World Health Organization. (2018). Fact sheet: Physical Activity.

World Health Organization. (2017). Health literacy. The solid facts: Health.

World Health Organization. (2013). Nutrition, Physical Activity and Obesity: Lithuania.

Wu, A. D., Begoray, D. L., MacDonald, M., Wharf Higgins, J., Frankish, J., Kwan, B., ... \& Rootman, I. (2010). Developing and evaluating a relevant and feasible instrument for measuring health literacy of Canadian high school students. Health Promotion International, 25(4), 444-452.

Zarcadoolas, C., Pleasant, A., \& Greer, D. S. (2005). Understanding health literacy: An expanded model. Health Promotion International, 20(2), 195-203. 